\title{
Moiré bands in transitional metal dichalcogenides: continuous Mott transition, quantum anomalous Hall and more.
}

\section{Quantum criticality in twisted transition metal dichalcogenides} Authors: Augusto Ghiotto, En-Min Shih, Giancarlo S. S. G. Pereira, Daniel A. Rhodes, Bumho Kim, Jiawei Zang, Andrew J. Millis, Kenji Watanabe, Takashi Taniguchi, James C. Hone, Lei Wang, Cory R. Dean; and Abhay N. Pasupathy Nature, 597, 345-349 (2021)

2. Continuous Mott transition in semiconductor moiré superlatticess Authors: Tingxin Li, Shengwei Jiang, Lizhong Li, Yang Zhang, Kaifei Kang, Jiacheng Zhu, Kenji Watanabe, Takashi Taniguchi, Debanjan Chowdhury, Liang Fu, Jie Shan, Kin Fai Mak

Nature 597, 350-354 (2021)

\section{Quantum anomalous Hall effect from intertwined moiré bands}

Authors: Tingxin Li, Shengwei Jiang, Bowen Shen, Yang Zhang, Lizhong Li, Trithep Devakul, Kenji Watanabe, Takashi Taniguchi, Liang Fu, Jie Shan, Kin Fai Mak https://arxiv.org/abs/2107.01796

\section{Recommended with a Commentary by Patrick A. Lee, MIT}

The transition metal dichalcogenide (TMD) familiy consists of a triangular lattice of 3d, $4 \mathrm{~d}$ or $5 \mathrm{~d}$ transition metal sandwiched between two triangular layers of chalcogen atoms (S, Se, Te). The resulting layered structure has served for 6 decades as excellent model compounds of 2D materials. In the 1960's and 70's, TMDs were intensively studied for harboring charge density wave $\left(\mathrm{TaS}_{2}, \mathrm{TaSe}_{2}\right.$ etc) and relatively high temperature superconductors $\left(\mathrm{NbSe}_{2}\right)$. More recently attention has been drawn to the $4 \mathrm{~d}$ and $5 \mathrm{~d}$ materials with their strong spinorbit coupling, resulting in Ising superconductor [1], topological insulators and topological superconductors $\left(W T e_{2}\right)[2]$. The weak van der Waals bonding between the sandwich layers makes them easy to cleave and intercalate, and the scotch-tape method (now known as the mechanical exfoliation technique) was widely applied 6 decades ago to make thin flakes. In the original paper of Geim and Novoselov, TMD monolayers were featured along with graphene. Graphene has stolen the show since that time and the recent development of twisted graphene layer continues to fuel the excitement. Now the time is ripe for TMD to emerge from the shadow and show its stuff, following the theoretical proposal of Wu et al.[3] 


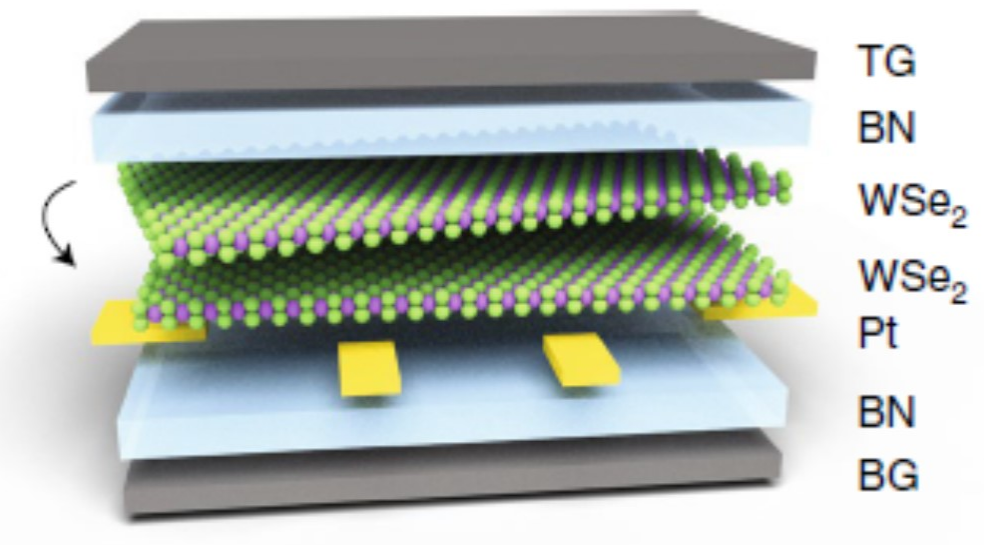

Figure 1: Illustration of homobilayer twisted TMD. From [4].

There are two ways to form bilayer moiré structures. First is the homobilayer where two layers of the same TMDs are twisted relative to each other, just like twisted bilayer graphene. [4] The second is heterobilayer, where two TMD with lattice constant mismatch are stacked on top of each other. [5] Both types are represented in the papers recommended above. Paper 1 studies twisted $\mathrm{WSe}_{2}$ while papers 2 and 3 study stacking of $\mathrm{MoTe}_{2}$ on top of $W \mathrm{We}_{2}$, albeit with different orientations, to be explained later. The resulting moiré unit cell typically has a unit cell size of $5-10 \mathrm{~nm}$, a bit smaller than magic angle graphene. In order to produce a well ordered structure in such a large unit cell, graphene has a key advantage in that the carbon bond is extremely strong, and both structural and impurity defects in the layers are minimal. Thus it is remarkable that apparently coherent flat bands also form for the less ideal case of TMDs. Nevertheless, the effect of disorder should be kept in mind. It is also worth noting that in the case of moiré TMD, the bandwidth varies smoothly with angle, and no magic angle is required.

There is an important difference between graphene moiré and TMD moiré that I would like to emphasize from the beginning. An important feature of twisted bilayer graphene is that in addition to spin, the band has valley degeneracy to a very good approximation and the two layers are relatively weakly hybridized. As a result it takes 8 electrons to completely fill the flat bands. The many degrees of freedom are the source of great complication or profound richness, depending on your point of view. In contrast, for the TMDs in question the low energy physics can be described by a doubly degenerate band and is filled by 2 electrons. This is because strong spin-orbit coupling locks the spin and valley, resulting in a single pseudo-spin degree of freedom. Furthermore, in the case of twisted TMD, the hybridization between layers is strong enough to split them and in the case of heterobilayer, the band off-set is large enough that the band is dominated by one of the layers. As a result, we have a single narrow band Hubbard model on a triangular (moiré) lattice, a dream come true for the strong correlation junkies among us. However, it is good to remember that the pseudo-spin degeneracy is not rotational invariant, unlike the standard Hubbard model.

A great advantage that these bilayer systems shares with twisted graphene is that by tuning the bottom and top gates separately, it is possible to tune the chemical potential to fix the system at a given doping and control the perpendicular electric field between the 

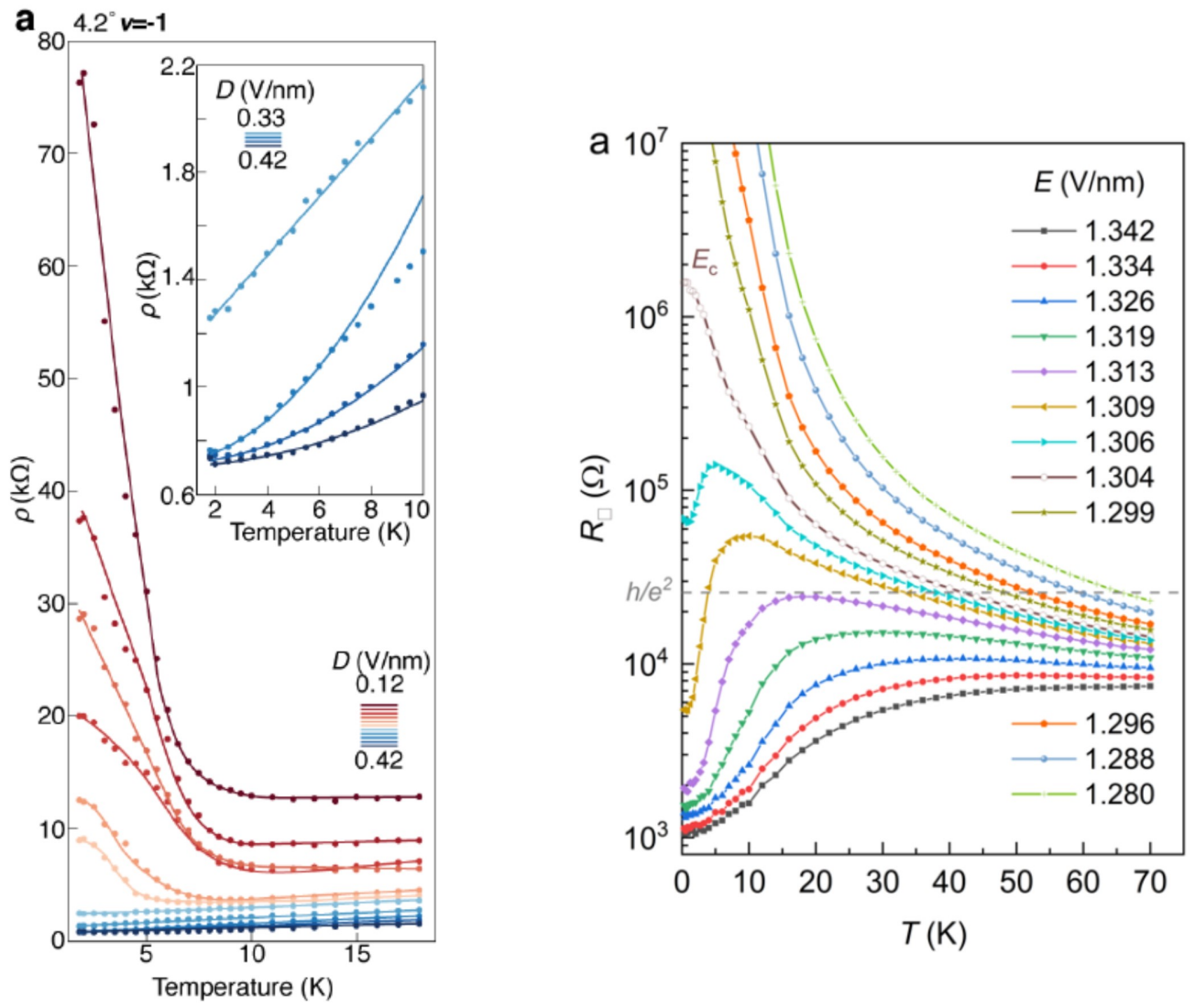

Figure 2: (a) Temperature dependence of the sheet resistance per square from paper 1, Ghiotto et al. in twisted $W S e_{2}$, for varying electric field D. Inset shows the change from quadratic to linear $\mathrm{T}$ dependence by zooming in to the lowest 4 plots in the main figure. (b) Similar plot from paper 2, Li et al in the heterobilayer $M o T e_{2} / W S e_{2}$. The critical electrical field is identified as the last plot that extrapolates to a finite resistance at low temperature. As $E_{c}$ is approached from the metallic side, the resistance goes as $T^{2}$ with a divergent coefficient and shrinking region of validity.

layers, therefore modifying the band parameters. This is called bandwidth controlled, as opposed to the more common doping control by gate. This flexibility has been exploited to great effect in the three recommended papers. In 1) and 2) the system is fixed to be at half filling and by tuning the electric field, it is possible to drive the system from a Mott insulator to a metal. In addition, paper 1) also explored the doping-controlled transition. A remarkable discovery is that the transition is continuous. There are a number of solid state systems where such a transition is driven by varying a continuous parameter such as pressure, but in almost all cases the transition is first order. In the early days Mott argued that the transition must be first order phase because the coulomb interaction jumps from undergoing metallic to dielectric screening across the transition. Here the nearby gates screen the long range Coulomb interaction due to the large carrier spacing and this old argument may not apply. Another thought is that in addition to the vanishing of the charge gap at the transition, the system typically shows magnetic ordering on the insulating side and it 
is difficult for two kinds of orders to vanish simultaneously, at least in the Landau picture. Thus a continuous transition is unexpected, even though it is clearly seen in plots of the sheet resistance per square vs. temperature for a variety of electric field reproduced in Fig. 2 from Paper 1 and 2.
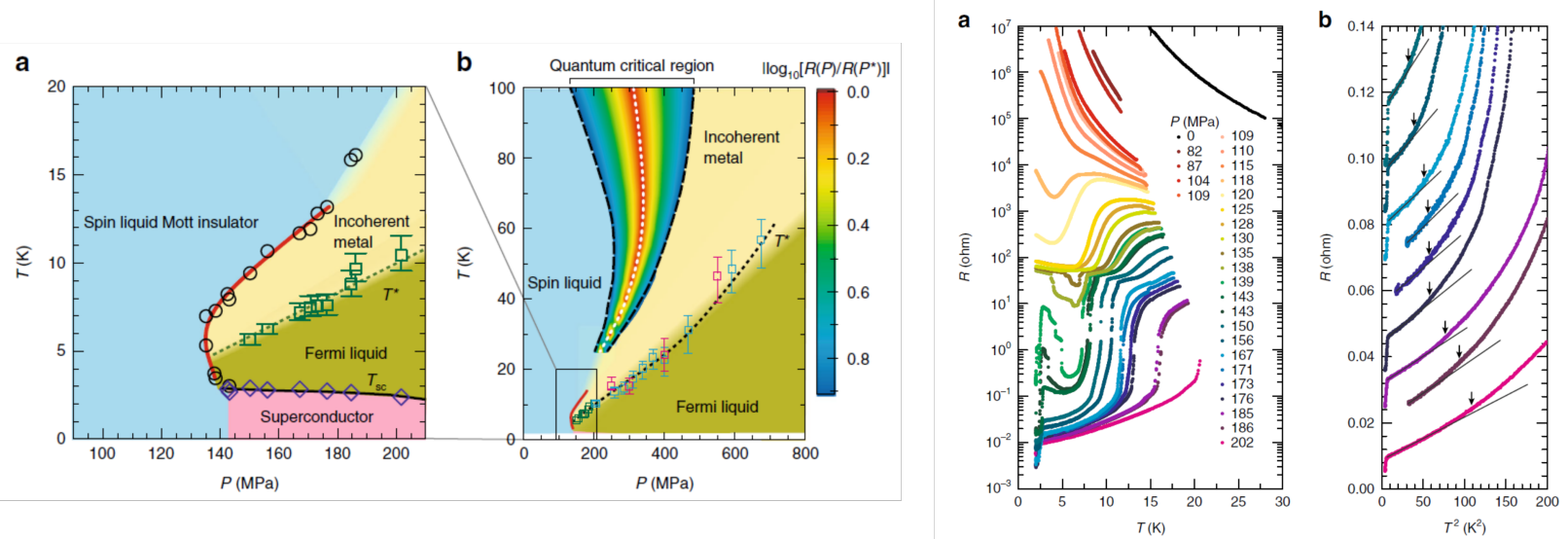

Figure 3: From Furukawa et al.[6] Left two figures show the phase diagram of $\kappa-$ $(E T)_{2} C u_{2}(C N)_{3}$ under pressure. The spin liquid Mott insulator transforms continuously to a $2.5 \mathrm{~K}$ superconductor and a metal. (a) is the zoom-in of (b). Right two figures show the resistance as a function of temperature for a range of pressure. As shown in (b) which is the zoom-in to the bottom 8 pressures in (a), the resistance scales as $T^{2}$ near the transition.

Prior to the current papers, the famous case of a claimed continuous transition is the organic system $\kappa-(E T)_{2} C u_{2}(C N)_{3}$. [6] The organic molecules $(E T)_{2}$ sit on an almost triangular lattice and is believed to be well described by a one band Hubbard model. $\kappa-$ $(E T)_{2} C u_{2}(C N)_{3}$ is a spin liquid candidate because no magnetic order has been found down to $\mathrm{mK}$ scale. Under pressure it becomes a $2.5 \mathrm{~K}$ superconductor, but the transition to the metal above this temperature has been analyzed to be continuous. The phase diagram is shown in Fig 3. One interesting feature is that the resistivity is linear in $T^{2}$ as long as the system is on the metallic side, even though superconductivity intervenes and it is not possible to go below 2.5K. (Right panel in fig. 3) For the $M o T e_{2} / W S e_{2}$ heterobilayer, the resistivity is also $T^{2}$, with a coefficient which diverges like a power law as the critical point is approached. (Fig. 2b) This is also the case for the twisted $W \mathrm{Se}_{2}$ homobilayer, with the added feature that the resistivity becomes linear in $\mathrm{T}$ at the transition point. (Fig 2a inset.) Part of the difference may have to do with the identification of the critical electric field. The critical resistance in fig $2 \mathrm{a}$ would have been $20 \mathrm{k}$ ohm instead of $1.2 \mathrm{k} \mathrm{ohm}$ if the criterion in fig. $2 \mathrm{~b}$ (described in the caption) were adopted. A possible source of this difference may have to do with a predicted jump in the resistance at the critical point to be discussed below. It is worth noting that the resistance at the critical electric field differs by two orders of magnitude between Fig $2 \mathrm{a}$ and $\mathrm{b}$, which may indicate stronger disorder in the $M o T e_{2} / W S_{2}$ heterobilayer. There are other differences in the temperature dependence of the three systems so that detailed interpretation of the data remains to be sorted out and disorder may play a role.

There is a theory of the continuous transition between a quantum spin liquid Mott 
insulator and a metal due to Senthil.[7] The spin liquid is assumed to have a spinon Fermi surface. In this case a continuous transition can happen naturally if the electron Fermi surface at half filling on the metallic side continuously loses its spectral weight and morphs into a spinon Fermi surface which has the same shape and area. A remarkable prediction of this theory is that in the zero temperature limit, the resistance at the transition has a sudden jump from a finite value on the metallic side, before going to infinity on the insulator side. The jump is a universal number times $h / e^{2}$. Given the fact that the data are still at a rather high temperature in all three cases, the jump, if it exists, is a bit hard to analyze. One might discern a jump of about $1 / 4 h / e^{2}$ between the blue and yellow curves in fig. 2a. A similar size jump would be too small to be visible in fig. $2 \mathrm{~b}$.

It should also be pointed out that while early specific heat data support a spinon Fermi surface in $\kappa-(E T)_{2} C u_{2}(C N)_{3}$ [8], thermal transport appears to show a gap [9] and recent electron spin resonance data shows conclusively that a spin gap develops below about 5K [10]. So Senthil's model may not apply to the organics, at least not down to low temperatures. On the other hand, this raises an interesting question: does the continuous Mott transition reported in papers 1 and 2 indicate that a spin liquid, possibly with a spinon Fermi surface, exists in the TMD moiré system? The first criterion is whether there is a magnetic phase transition. Normally it is close to impossible to measure the magnetic susceptibility in a monolayer, but here nature brings yet another gift. In the TMD the spin-momentum locking at the $\mathrm{K}$ point leads to selection rules and the phenomenon of magnetic circular dichroism, so that the magnetization can be measured optically. The spin susceptibility is reported in paper 2). It shows a Curie Weiss behavior at high temperature which evolves smoothly through the Mott transition. The Weiss temperature is $30-40 \mathrm{~K}$, but There is no kink at any temperature which may be associated with antiferromagnetic ordering down to $1.7 \mathrm{~K}$. This gives encouragement to the notion that the state just on the insulator side may be a spin liquid which may have a spinon Fermi surface. Indeed, proximity to the Mott transition has been suggested as a route to quantum spin liquid many years ago. [11, 12]

Spin liquids are notoriously difficult to verify experimentally, especially in monolayer systems where the small sample size precludes many of the traditional tools such as specific heat, NMR etc. Recently progress has been made using STM to probe another spin liquid candidate monolayer $1 T-\mathrm{TaSe}_{2}$, which also happens to be a TMD. [13] A well known "star of David" charge ordering expands the unit cell to 13 sites. The band is flat in the reduced Brillouin zone, giving enough correlation to drive the system to a Mott insulator. So this can be considered the baby version of the TMD moiré which has a much larger unit cell. Interestingly, a related material, bulk $1 T-T a S_{2}$ is also an insulator that undergoes an apparently continuous transition to a metal at a very modest pressure of 0.8 GP. [14, 15] So these systems are also proximate to a insulator to metal transition. Unfortunately, in the moiré system a top gate is needed, which precludes the use of STM. It will be interesting to attempt tunneling spectroscopy in a non-contact way by modulating the capacitance [16], or even obtain momentum dependent dispersion using a recently developed capacitance tunneling technique. [17]

It has been proposed that the twisted TMD bands may have nontrivial topology of the Kane-Mele type [18]. However in the heterobilayer system with AA stacking as in paper 2 , nontrivial topology is not expected. On the other hand, if the layers are stacked with a 60 degree rotation, forming an $\mathrm{AB}$ stacking, paper (3) reports the discovery that at half 
filling, the ground state has a Chern number and exhibit quantum anomalous Hall effect. It is argued that a sufficiently strong interlayer electric field may create a band structure with opposing Chern numbers [19] or lead to band inversion [20]. Another suggestion is that strain from the lattice relaxation gives rise to gauge fields which drives the nontrivial topology [21].

With hundreds of compounds at our disposal, a myriad of stacking combinations is possible. It seems likely that we are only beginning to scratch the surface of the moiré TMD system and moiré surprises will keep coming.

\section{References}

[1] J. Lu et al, Science 350, 1353 (2015).

[2] V. Fatemi et al, Science 362, 926 (2018).

[3] F. Wu et al, PRL 121, 026402 (2018).

[4] L. Wang et al, Nature materials 19, 861 (2020).

[5] Y. Tang et al, Nature 579, 353 (2020).

[6] T. Furukawa et al, Nature Comm. 9, 307 (2018).

[7] T. Senthil, PRB 78, 045109 (2008). Phys. Rev. B 74, 134512 (2006).

[8] Y. Shimizu et al PRL 91, 107001 (2003).

[9] M. Yamashita et al, Science 328, 1246 (2010).

[10] Miksch et al., Science 372, 276 (2021).

[11] O. Motrunich Phys. Rev. B 72, 045105 (2005).

[12] S.-S. Lee et al, Phys. Rev. Lett. 95, 036403 (2005).

[13] W. Ruan et al, Nature physics (2021) https://doi.org/10.1038/s41567-021-01321-0.

[14] B. Sipos et al, Nature Materials 7, 960 (2008).

[15] T. Ritschel et al Physical Review B 87, 125135 (2013).

[16] J. Jang et al, Nature Phys 13, 340-344 (2017).

[17] J. Jang et al, Science 358, 901 (2017).

[18] F. Wu et al PRL 122, 086402 (2019).

[19] Y. Zhang et al, arXiv: 2107.02167.

[20] T, Devakul and L. Fu, arXiv to be posted.

[21] Y. Xie et al, arXiv: 2106.13991. 